Synthèse - Review

\title{
Les capacités d'adaptation du silure glane en hypoxie: un cas exemplaire d'homéostasie du milieu intérieur
}

\author{
Jean-Charles Massabuau et Jean Forgue \\ Laboratoire de Neurobiologie et Physiologie Comparées, Universilé de Bordeaux I-CNRS, \\ place du Dr Peyneau, 33120 Arcachon, France.
}

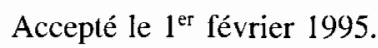

Massabuau J. C., J. Forgue. Aquat. Living Resour., 1995, 8, 423-430.

How sheatfish, Silurus glanis, maintains oxygen supply in hypoxia: a key exumple of oxygen homeostasis.

Abstract

How oxygen is supplied independently of water oxygenation in water breathers is an age-old problem of great interest to fish farmers, ecologists and fish physiologists alike. This review paper (i) summarizes the strategy used by the sheatfish Silurus glanis to deal with this problem and also (ii) considers the case of $S$. glanis within a general framework that explains how $\mathrm{O}_{2}$ is supplied in many water breathers. One of the basic concepts that we have elaborated during the past 10 years, partly thanks to the sheatfish model, deals with the notion of $\mathrm{O}_{2}$ homeostasis in the internal medium. We demonstrated that, in a wide range of physiologically different water-breathers under resting conditions, a low arterial $\mathrm{PO}_{2}$ ( $1-3 \mathrm{kPa}$ at $13-15^{\circ} \mathrm{C} ; 1 \mathrm{kPa}=7.5 \mathrm{~mm} \mathrm{Hg}$ ) set most of the time just above the arterial $\mathrm{Po}_{2}$ at the anaerobic threshold (0.7-1.2 kPa), provides a sufficient pressure head to ensure an adequate $\mathrm{O}_{2}$ supply at the cellular level. This is largely independent of $\mathrm{Po}_{2}$ changes in the external medium in the range 3-40 kPa (i.e. 1.5-20 mg.1 $1^{-1}$ at $13^{\circ} \mathrm{C}$ ). The sheatfish Silurus glanis has a resting $\mathrm{O}_{2}$ consumption that is quite comparable to values measured in teleosts like the carp, cel or tench and in crustaceans and is approximately half of the consumption reported for salmonids. The sheatfish is able to maintain this $\mathrm{O}_{2}$ consumption constant for at least $24 \mathrm{~h}$ down to a critical inspired $\mathrm{Po}_{2}=2-3 \mathrm{kPa}\left(1-1.5 \mathrm{mg} \cdot \mathrm{l}^{-1}\right.$ at $\left.13^{\circ} \mathrm{C}\right)$. Ventilatory flow rate plays a fundamental role in this maintenance while the inspired $\mathrm{Po}_{2}$ varies in the external medium. Indeed, ventilation increases in hypoxic water and decreases in hyperoxic water while the blood flow rate remains constant. The main result of this ventilatory adaptation is the constancy of the arterial $\mathrm{Po}_{2}$ at $2 \mathrm{kPa}$, that is maintained independently of the inspired $\mathrm{Po}_{2}$ in the range $3-40 \mathrm{kPa}$ (i.e. $1.5-20 \mathrm{mg} .1^{-1}$ at $13^{\circ} \mathrm{C}$ ). In this inspired $\mathrm{PO}_{2}$ range the blood $\mathrm{pH}$ and the blood $\mathrm{O}_{2}$ content also remain constant. Consequently, in terms of $\mathrm{O}_{2}$, the homeostasis of the internal medium is fulfilled.

Although we have not studied these mechanisms in Silurus at temperatures other than $13^{\circ} \mathrm{C}$, for temperature changes in the range $10-23^{\circ} \mathrm{C}$ at least, it is reasonable to assume that the arterial $\mathrm{Po}_{2}$ and the critical water $\mathrm{Po}_{2}$ over which $\mathrm{O}_{2}$ consumption can be maintained constant probably remains steady. This conclusion is based on a comparison with reported data in the literature which cover a large range of physiologically different water-breathers. First, in the carp Cyprinus carpio, the crayfish Astacus leptodactylus and the crab Carcinus maenas, the arterial $\mathrm{Po}_{2}$ remains in the same low range whatever the temperature change from $10-13^{\circ} \mathrm{C}$ to $23-24.5^{\circ} \mathrm{C}$. Second, in the carp Cyprinus carpio, the trout Oncorhynchus mykiss and the crayfish Astacus leptodactylus, the resting $\mathrm{O}_{2}$ consumption can be maintained constant down to the same critical $\mathrm{Po}_{2}(3 \mathrm{kPa})$, independently of the temperature from $10-13$ to $20-23^{\circ} \mathrm{C}$.

It is concluded that the sheatfish Silurus glanis at rest has a substantial capacity for withstanding prolonged periods of hypoxia down to $\mathrm{Po}_{2}=2-3 \mathrm{kPa}\left(1-1.5 \mathrm{mg} . \mathrm{l}^{-1}\right.$ depending on the temperature). However, we stress that currently we have no idea of this potential in animals reared in intensive fish farms where gills could be physiologically altered or other pathological disorders could have developed. 
Keywords: Silurus glanis, fish farming, ecology, oxygen, ventilation, blood, hypoxia, normoxia, hyperoxia, hemoglobin, blood $\mathrm{pH}$.

Résumé

Nous étudions les principes généraux qui expliquent comment les animaux aquatiques assurent leurs ćchanges gazeux $\left(\mathrm{O}_{2}\right.$ et $\left.\mathrm{CO}_{2}\right)$ en fonction d'une part, de leurs besoins physiologiques et d'autre part, des changements des caractéristiques physico-chimiques du milieu. Cette synthèse, basée sur le cas exemplaire du silure glane, résume nos observations sur les mécanismes qui permettent à des animaux aquatiques physiologiquement très différents de s’adapter aux variations d'oxygénation du milieu ambiant. Le but de la stratégie d'adaptation est de maintenir constant l'ćtat d'oxygénation du sang, à une faible valeur de pression partielle d' $\mathrm{O}_{2}\left(\mathrm{PO}_{2}\right)$ dans le sang artériel, indépendamment des variations de $\mathrm{Po}_{2}$ dans l'eau. Cher. le silure, la valeur la plus couramment mesurée de $\mathrm{Po}_{2}$ dans le sang artériel est $2 \mathrm{kPa}(15 \mathrm{~mm} \mathrm{Hg})$ pour des variations de $\mathrm{PO}_{2}$ dans l'eau comprises entre 3 et $40 \mathrm{kPa}\left(1,5\right.$ à $20 \mathrm{mg} \cdot \mathrm{l}^{1}$ à $\left.13^{\circ} \mathrm{C}\right)$. Ce qui est aussi particulier cher cet animal est que toute l'adaptation est réalisée par la ventilation: il n'y a aucun changement de débit cardiaque, ni de modification de l'aftinité de l'hémoglobine pour l'oxygène via le $\mathrm{pH}$ sanguin. $\mathrm{La}$ valeur de $\mathrm{Po}_{2}$ inspirée jusqu’à laquelle la consommation d' $\mathrm{O}_{2}$ peut être assuréc pendant au moins $24 \mathrm{~h}$ à $13^{\circ} \mathrm{C}$ cst 2 à $3 \mathrm{kPa}$. Nous pensons que che $z$ le silure cette valeur de $\mathrm{Po}_{2}$ est largement indépendante de la température car chez des animaux aussi divers que la carpe, la truite ou l'ćcrevisse (adaptés au laboratoire à différentes températures comprises entre 10 et $23^{\circ} \mathrm{C}$ ) la valeur de $\mathrm{Po}_{2}$ critique est aussi égale à $2-3 \mathrm{kPa}$ indépendamment de la température. Toutes les mesures que nous citons ont été faites chez des animaux à jeun au laboratoire mais le principe du "fonctionnement » de très nombreux animaux aquatiques avec de faibles valeurs de $\mathrm{Po}_{2}$ artériel, indépendamment de la température, a été vérifié dans le milieu naturel. Nous n'avons actuellement aucune idée sur les capacités du silure dans un élevage intensif, en particulier lorsque des pathologies s'y développent. Néanmoins, il est clair que les capacités maximales d'adaptation du silure face à l'hypoxie sont très grandes.

Mots-clés : Silurus glanis, aquaculturc, écologie, oxygène, ventilation, sang, hypoxie, normoxie, hyperoxie, $\mathrm{pH}$ sanguin.

\section{INTRODUCTION}

La question de l'approvisionnement en oxygène chez les animaux aquatiques est un problème qui intéresse aussi bien les professionnels de l'aquaculture, que les spécialistes de l'environnement ou les chercheurs fondamentalistes. Il s'agit de savoir pour les premiers, comment éviter des mortalités et favoriser la croissance; pour les seconds, comment interpréter des observations parfois contradictoires dans le milieu naturel; et enfin pour les derniers, de comprendre les mécanismes intimes qui permettent d'assurer le transfert de l'oxygène du milieu ambiant jusqu'à la cellule.

Cette synthèse est centrée sur les mécanismes physiologiques mis en jeu chez le silure glane, Silurus glanis, pour faire face aux problèmes d'hypoxie dans l'eau d'après les données de Forgue et al. (1989). En bref, la stratégie utilisée est basée sur le maintien d'une pression partielle d' $\mathrm{O}_{2}\left(\mathrm{Po}_{2}\right)$ dans le sang artériel à une valeur qui est le plus couramment faible et constante ( $2 \mathrm{kPa} \approx 15 \mathrm{~mm} \mathrm{Hg}$ ). Cette valeur est indépendante des variations de $\mathrm{Po}_{2}$ dans l'eau entre 3 et $40 \mathrm{kPa}$ $\left(1,5\right.$ et $20 \mathrm{mg} \cdot \mathrm{l}^{-1}$ à $\left.15^{\circ} \mathrm{C}\right)$. Pour rappel, une eau ćquilibrée à l'air à $15^{\circ} \mathrm{C}$, contient $10 \mathrm{mg} . \mathrm{l}^{-1}$ d'oxygène à une pression partielle de $21 \mathrm{kPa}$ ou $160 \mathrm{~mm} \mathrm{Hg}$. Le résultat de cette «stratégic physiologique» est une parfaite homéostasie du milieu intérieur en terme d'oxygénation et une indépendance extrême vis-à-vis des conditions d'oxygénation de l'eau.

À l'origine, cette stratégie a été observée pour la première fois chez un crustacé, l'écrevisse Astacus leptodactylus (Massabuau et Burtin, 1984) puis ensuite chez un mollusque, la moule d'eau douce Anodonta cygnea (Massabuau et al., 1991). Le silure, l'écrevisse et l'anodonte n'avaient pas été choisis par hasard mais comme représentant des trois grands groupes d'animaux aquatiques que sont les poissons téléostéens, les crustacés et les mollusques. Aujourd'hui, on sait que de faibles $\mathrm{PO}_{2}$ artériels en normoxie - qui présentent l'avantage de pouvoir être maintenus en hypoxie - se rencontrent chez de très nombreuses espèces indépendamment du phylum, du milieu de vie (eau douce ou salée), de la saison, de l'organisation du système respiratoire (type de branchie, de pompe ventilatoire, de système circulatoire qui peut être clos ou ouvert), de la présence ou de l'absence de pigment respiratoire et, lorsqu'il est présent, de la concentration de pigment respiratoire et de son affinité pour l'oxygène dans une gamme de $\mathrm{P}_{50}$ variant de 0,2 à $2 \mathrm{kPa}$ (Forgue et al., 1992).

On a donc là un concept nouveau qui permet d'aborder les problèmes d'oxygène dans le milieu aquatique d'une façon très globale (sans qu'il soit en aucun cas question de nier les différences entre espèces). C'est dans ce cadre que nous proposerons parfois des extrapolations que nous pensons applicables au silure alors que les résultats expérimentaux ont été obtenus chez d'autres animaux.

\section{Rappel de physiologie respiratoire}

La fonction respiratoire regroupe l'ensemble des mécanismes qui assurent le transfert d' $\mathrm{O}_{2}$ et de $\mathrm{CO}_{2}$ entre le milieu ambiant et les cellules où l'oxygène est 
consommé et le gaz carbonique produit. Pour assurer à tout moment cet approvisionnement en oxygène, deux conditions doivent être simultanément remplies au niveau du sang artériel. D'une part, il faut maintenir des gradients de pression suffisamment élevés de façon à ce que l'oxygène puisse pénétrer dans les cellules. D'autre part, il faut apporter une quantité suffisante de molécules d'oxygène de façon à ce que le sang puisse jouer son rôle de réservoir (à ce niveau, le pigment respiratoire - hémoglobine ou hémocyanine par exemple - joue un rôle fondamental puisqu'il permet d'augmenter considérablement la capacité de transport du sang par rapport à l'eau). Le système qui assure cette fonction de transfert est formé d'une succession de compartiments disposés en série. En accord avec Dejours (1981), on peut résumer cette succession en deux étapes de convection et deux étapes de diffusion qui sont représentées schématiquement sur la figure 1.

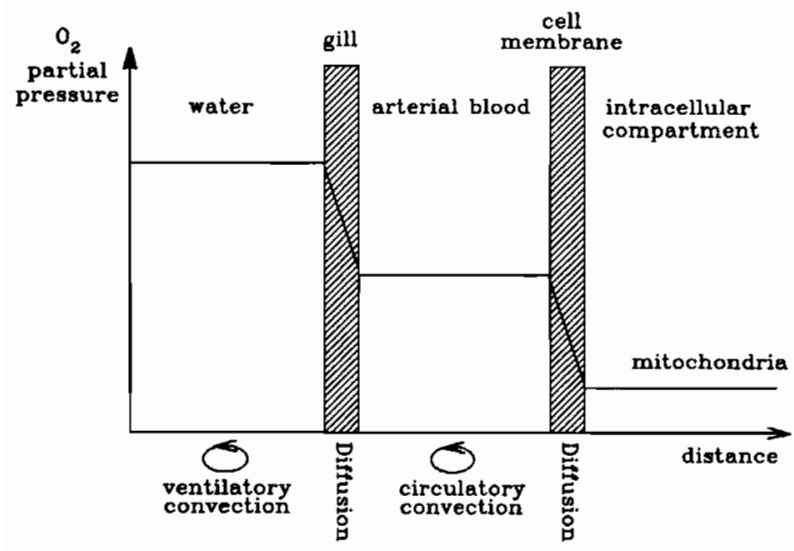

Figure 1. - Schéma de principe de la cascade de pression particlle d'oxygène $\left(\mathrm{PO}_{2}\right)$ depuis l'eau inspirée jusqu'au milieu intracellulaire et la mitochondrie chez un animal aquatique. Les barrières de diffusion sont représentées en grisé (d'après Dejours, 1981).

Diagram illustrating the changes of $\mathrm{O}_{2}$ partial pressures $\left(\mathrm{PO}_{2}\right)$ which occur en route from inspired water until cell and mitochondria in a water-breather. The gas exchange system is composed of a set of serially linked compartments that give rise to a four-step model through which $\mathrm{O}_{2}$ and $\mathrm{CO}_{2}$ are transferred by convection and diffusion. During the first step the water is moved by ventilatory convection. The second step involves diffusion: $\mathrm{O}_{2}$ diffuses through the gill epithelium the pressure difference between $\mathrm{Po}_{2}$ in the water and gill capillary blood. The gill status is fundamental here: if the epithelium becomes thicker, the diffusive resistance increases and the gas diffusion becomes limited. The third step is blood convection: starting from the gills, the blood transports $\mathrm{O}_{2}$ to the cell through arteries and arterial capillaries. The fourth step is diffusive again: $\mathrm{O}_{2}$ diffuses from the arterial capillaries into the cell under the difference between $\mathrm{Po}_{2}$ in the arterial blood and $\mathrm{PO}_{2}$ in the intracellular medium (from Dejours, 1981).

- La $1^{\text {re }}$ étape est la ventilation. Il s'agit de renouveler par convection l'eau au contact des branchies.

- La $2^{\mathfrak{c}}$ étape est la diffusion des gaz à travers la paroi des branchies, de l'eau vers le sang pour
$\mathrm{O}_{2}$ et vice-versa pour le $\mathrm{CO}_{2}$. L'état des branchies est ici fondamental: en particulier, un épaississement de l'épithélium branchial augmentera la résistance diffusive.

- La $3^{\text {e }}$ étape est celle de la circulation sanguine. C'est à nouveau une étape convective. Le sang artériel enrichi en $\mathrm{O}_{2}$, fixé ou non sur un pigment respiratoire, va transporter l'oxygène via les artères et les capillaires artériels - depuis les branchies jusqu'aux cellules. Il revient ensuite vers les branchies, chargé en $\mathrm{CO}_{2}$.

- La $4^{e}$ étape est à nouveau une étape de diffusion. L'oxygène va maintenant devoir traverser la paroi des capillaires jusqu'à l'intérieur de la cellule où il sera consommé. Le gaz carbonique suit le trajet inverse.

\section{Quels sont les besoins en oxygène du silure glane?}

Ou, en gardant en mémoire les différentes étapes résumées ci-dessus, quelle est la quantité d'oxygène qui va devoir être transportée par unité de temps de l'eau inspirée jusqu'à la cellule ? En fait, à température égale, ces besoins sont du même ordre de grandeur que ceux des autres animaux aquatiques dont le comportement est comparable (tabl. 1). Ils sont en particulier semblables à ceux de la carpe (Cyprinus carpio), de l'anguille (Anguilla anguilla) ou de la tanche (Tinca tinca) mais environ deux fois inférieurs à ce qui a été rapporté pour des poissons beaucoup plus actifs comme la truite (Oncorhynchus mykiss) et le saumon (Salmo salar). À noter aussi qu'ils sont identiques à ceux des crustacés (écrevisses Astacus leptodactylus et crabes).

Tableau 1. - Valeurs de consommation d'oxygène ( $\dot{\mathrm{Mo}}_{2}, \bar{x} \pm$ S.E.) chez quelques espèces d'animaux aquatiques.

Oxygen consumption values $\left(\dot{M} o_{2}, \bar{x} \pm S . E\right.$.) in various aquatic species.

\begin{tabular}{|c|c|c|c|}
\hline Espèce & $\begin{array}{c}\mathrm{T} \\
\left({ }^{\circ} \mathrm{C}\right)\end{array}$ & $\begin{array}{c}\dot{\mathrm{Mo}}_{2} \\
(\mu \mathrm{molmmin} \\
\left.. \mathrm{kg}^{-1}\right)\end{array}$ & Référence \\
\hline Silurus glanis & 13 & $15.4 \pm 1.5$ & Forgue et al. (1989) \\
\hline \multirow[t]{2}{*}{ Anguilla anguilla } & 11.5 & $16.8 \pm 0.8$ & $\begin{array}{l}\text { Kirsch et } \\
\text { Nonnotte (1977) }\end{array}$ \\
\hline & 18 & $25.6 \pm 0.8$ & $\begin{array}{l}\text { Le Moigne et al. } \\
(1986)\end{array}$ \\
\hline Cyprinus carpio & 15 & $27.8 \pm 3.8$ & Ott et al. (1980) \\
\hline Tinca tinca & 13 & $20.2 \pm 1.2$ & Nonnotte (1981) \\
\hline Oncorhynchus mykiss & 15 & $44.6 \pm 2.8$ & Ott et al. (1980) \\
\hline Salmo salar & 15 & $35.8 \pm 1.8$ & Maxime et al. (1990) \\
\hline Astacus leptodactylus & 13 & $13.8 \pm 0.8$ & $\begin{array}{l}\text { Massabuau } \\
\text { et Burtin (1984) }\end{array}$ \\
\hline
\end{tabular}

\section{Comment le silure glane assure son approvisionnement en oxygène quand le niveau d'oxygénation varie dans l'eau?}

Et tout d'abord, dans quelle gamme de pression partielle d'oxygène dans l'eau $\left(\mathrm{Pwo}_{2}\right)$, peut-il 
maintenir sa consommation d'oxygène constante? Nous avons abordé ce problème en travaillant à $13^{\circ} \mathrm{C}$ sur de jeunes animaux à jeun de 100 -150 g dont on sait que le métabolisme par unité de poids est supérieur à celui des adultes. Nous les avons exposés pendant des périodes de $24 \mathrm{~h}$ à différents niveaux fixes de $\mathrm{PwO}_{2}$ compris entre l'hyperoxie $\left(\mathrm{PWO}_{2}=40 \mathrm{kPa}\right.$, c'est-à-dire une concentration d'O dans l'eau, $\mathrm{CWO}_{2} \approx 20 \mathrm{mg} \cdot \mathrm{l}^{-1}$ ) et l'hypoxic sévère $\left(\mathrm{PwO}_{2}=3 \mathrm{kPa}\right.$, c'est-à-dire $\left.\mathrm{CwO}_{2} \approx 1,5 \mathrm{mg} \cdot \mathrm{I}^{-1}\right)$. Puis, à la fin de ces $24 \mathrm{~h}$, nous avons mesuré les valeurs de consommation d'oxygène. Nous avons adopté cette démarche de façon à être sûr de travailler dans des états stationnaires, loin des phases de déséquilibres qui sont liées aux périodes d'ajustement. Ce faisant, nous nous sommes intéressés aux résultats de l'ajustement (et aux possibilités d'ajustement) plutôt qu'aux événements survenant pendant les phases transitoires. La figure $2 \mathrm{~A}$ résume de façon très claire le comportement du silure dans ces conditions: de 20 à $1,5 \mathrm{mg} . \mathrm{l}^{-1}$ ( $40 \mathrm{à} 3 \mathrm{kPa}$ ), la consommation d'oxygène reste constantc et est totalement indépendante des variations d'oxygénation ambiante. D'après les quelques animaux où nous avons recherché la limite inférieure du phénomène, il semble même que la consommation d' $\mathrm{O}_{2}$ puisse être maintenue jusqu'à $\mathrm{Pwo}_{2}=2 \mathrm{kPa}\left(\mathrm{Cwo}_{2} \approx 1 \mathrm{mg} .1^{-1}\right.$ à $13{ }^{\circ} \mathrm{C}$ ) pendant au moins $24 \mathrm{~h}$.

Un point remarquable est l'évolution de la ventilation à ces différents niveaux d'oxygénation. Si on part de la valeur de référence en normoxie pour un poisson d'un kilogramme (50-60 ml.min '), on s'aperçoit que la ventilation augmente quand la quantité d'O $\mathrm{O}_{2}$ disponible dans l'eau diminue et qu'elle diminue dans les conditions inverses, c'est-à-dire en hyperoxic. $\grave{A} \mathrm{PwO}_{2}=3 \mathrm{kPa}\left(=1,5 \mathrm{mg} . \mathrm{l}^{-1}\right)$, le débit d'eau ventilée atteint en moyenne $400-450 \mathrm{ml} \cdot \mathrm{min}^{-1} \cdot \mathrm{kg}^{-1}$ alors qu'à $40 \mathrm{kPa}\left(\mathrm{Cwo}_{2} \approx 20 \mathrm{mg} \cdot \mathrm{l}^{-1}\right)$, il chute à $25 \mathrm{ml} \cdot \mathrm{min}^{-1} \cdot \mathrm{kg}^{-1}$ ( $f \mathrm{~g} .2 \mathrm{~B}$ ). Mais pendant ce temps, le débit circulatoire ne varie absolument pas. Il reste constamment égal à environ $10 \mathrm{ml} \cdot \mathrm{min}^{-1} \cdot \mathrm{kg}^{-1}$ ( fig. 2C). Pourquoi le débit sanguin ne change-til pas quand la quantité d'oxygène disponible dans l'eau diminue? La réponse est dans l'évolution des pressions partielles d'O $\mathrm{O}_{2}$ dans le sang. En fait, que ce soit dans le sang artériel où dans le sang veineux, les valeurs les plus couramment mesurées de $\mathrm{PO}_{2}$ ne changent pas statistiquement. Elles restent à un niveau faible et constant quel que soit $\mathrm{Po}_{2}$ dans l'eau inspirée: $\mathrm{PO}_{2}$ artériel $\approx 2 \mathrm{kPa}$ et $\mathrm{Po}_{2}$ veineux $\approx 0,5 \mathrm{kPa} ;$ fig. $2 \mathrm{D}$ ). Les valcurs sont faibles, mais tout à fait suffisantes en ce qui concerne $\mathrm{PO}_{2}$ artériel pour saturer pratiquement la totalité du pigment respiratoire: à $\mathrm{Po}_{2}$ artéricl $\approx 2 \mathrm{kPa}, 85 \% \mathrm{du}$ pigment est en effet saturé ( $f l g .3$ ). Maintenir en permanence des valeurs de $\mathrm{Po}_{2}$ artérielles supérieures ne serait pas très utile, même si par principe le gain en terme de quantité d'O $\mathrm{O}_{2}$ transportée ne peut pas être considéré comme négligeable. Cela devrait être fait en maintenant un débit ventilatoire supérieur
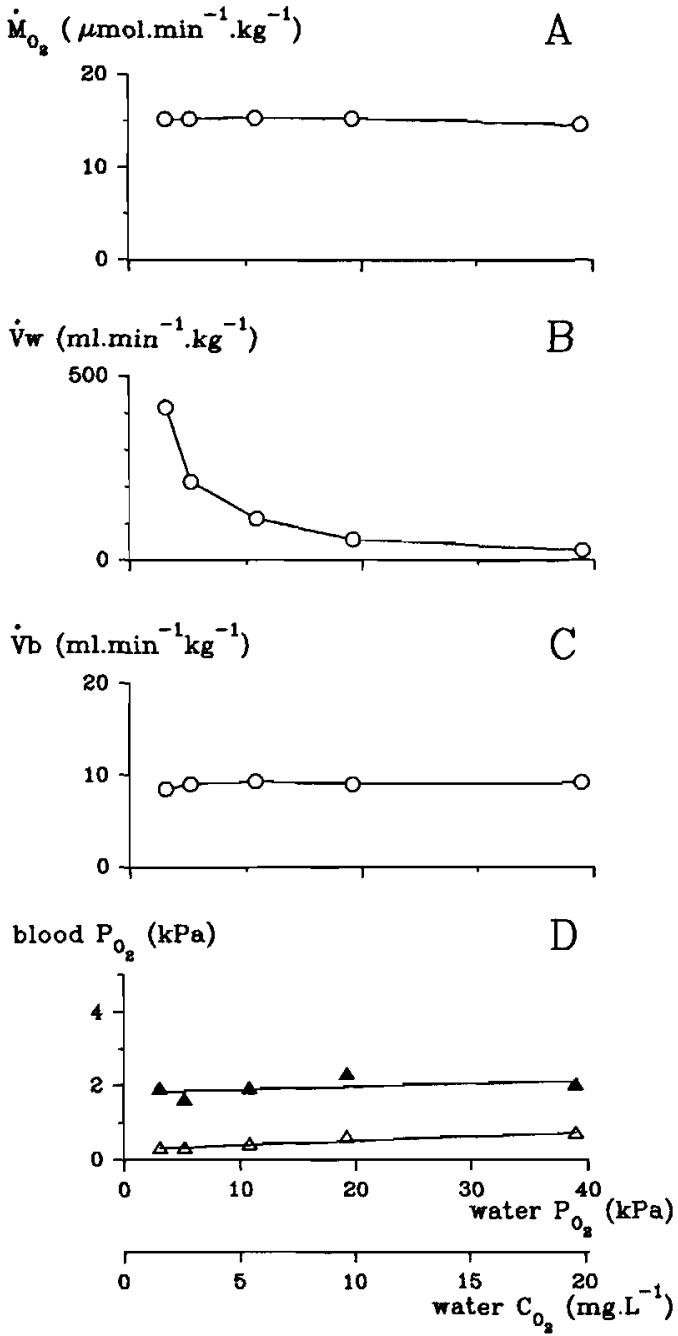

Figure 2. - Evolution: $\mathbf{A}$, de la consommation d' $\mathrm{O}_{2}\left(\mathrm{M}_{2}\right) ; \mathbf{B}$, du débit ventilatoire $\left(\dot{V}_{w}\right) ; \mathbf{C}$, du débit cardiaque $(\dot{V} b)$ et $\mathbf{D}$, des pressions partielles d' $\mathrm{O}_{2}$ dans le sang artéricl (symboles pleins) et le sang veineux (symboles ouverts) chez des silures glanes adaptés depuis $24 \mathrm{~h}$ à différentes valeurs de $\mathrm{Po}_{2}$ dans l'eau inspirée. $\mathrm{T}=13^{\circ} \mathrm{C}$ et $n=5$ à 7 animaux par point (d'après Forgue et al, 1989).

Steady-state values of $\mathbf{A}$, oxygen consumption $\left(\dot{M}_{2}\right)$; $\mathbf{B}$, ventilatory flow rate ( $(\vec{W}) ; \mathrm{C}$, blood flow rate $(\dot{V} b)$ and $\mathrm{D}, \mathrm{O}_{2}$ partial pressure in the arterial (closed symbols) and venous (open symbols) blood of Silurus glanis after $24 \mathrm{~h}$ exposure at selected and fixed $\mathrm{Po}_{2}$ in the inspired water. $T=13^{\circ} \mathrm{C}$ and $n=5-7$ fish. When the inspired $P_{0}$ varies between 40 and $3 \mathrm{kPa}(300)$ and $15 \mathrm{~mm} \mathrm{Hg}$ i.c. $C \mathrm{wo}_{2}=20$ and $\left.1.5 \mathrm{mg} . \mathrm{l}^{-1}\right)$, the key adaptation is a maintenance of oxygen consumption by ventilatory adjustment with no change in blood flow rate. At each inspired $\mathrm{PO}_{2}$, the most frequently measured arterial $\mathrm{Po}_{2}$ remains steady and low $(=2 \mathrm{kPa}$ or $15 \mathrm{~mm} \mathrm{Hg})$. Consequently, the $\mathrm{O}_{2}$ homeostasis of the internal medium is fulfilled independently of the external medium (from Forgue et al., 1989).

pour augmenter de façon permanente $\mathrm{Po}_{2}$ dans l'eau au niveau des branchies, et cela représenterait un coût énergétique supplémentaire. En tout cas, les mesures physiologiques et l'observation des animaux montrent que ces faibles valeurs de $\mathrm{Po}_{2}$ sanguines 


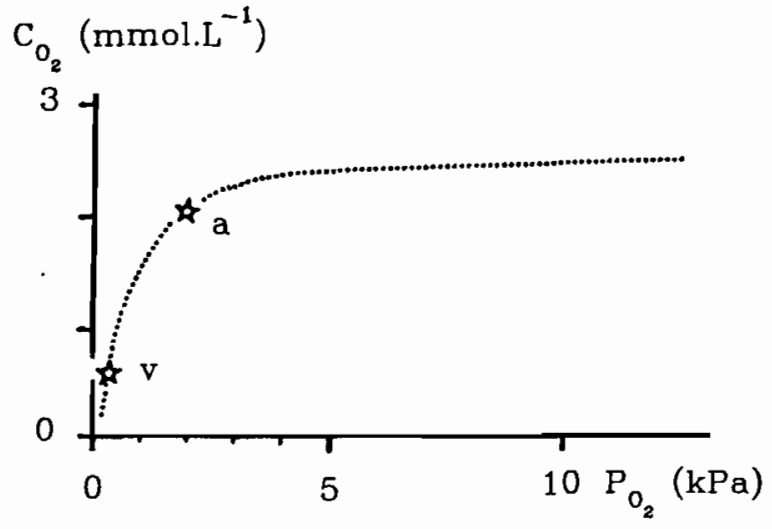

Figure 3. - Courbe de dissociation de l'hémoglobine du silure glane. La courbe a été déterminée in vitro pour des valeurs de $\mathrm{pH}(7,96)$ et d'hématocrite $(14 \%)$ physiologiques. Les valeurs de $\mathrm{Po}_{2}$ artériel (a) et de $\mathrm{PO}_{2}$ veineux (v) déterminées in vivo (fig. $2 \mathrm{D}$ ) sont reportées sur la courbe. $\mathrm{A} \mathrm{PO}_{2}$ artériel $=2 \mathrm{kPa}(15 \mathrm{~mm} \mathrm{Hg}), 85 \%$ du pigment respiratoire est déjà saturé (d'après Forgue et al., 1989).

In vitro $\mathrm{O}_{2}$ binding curve for Silurus at physiological blood $\mathrm{pH}$ (7.96) and hematocrit value (14\%). (a), arterial $\mathrm{Po}_{2} ;(v)$, venous $\mathrm{Po}_{2}$ (fig. $2 \mathrm{D})$. The in vivo measured arterial $\mathrm{Po}_{2}=2 \mathrm{kPa}(15 \mathrm{~mm} \mathrm{Hg})$ is large enough to saturate $85 \%$ of the respiratory pigment (from Forgue et al., 1989).

sont parfaitement suffisantes pour avoir des animaux en parfaite santé, extrêmement vigiles et agressifs!

Un autre paramètre fondamental, quand on analyse ces mécanismes, est l'évolution de l'état acide-base sanguin. En effet le pH du sang influence les caractéristiques des pigments respiratoires en diminuant ou en augmentant leur affinité (effet Bohr) et leur capacité de transport (effet Root) vis-à-vis de l'oxygène. Or quand un animal est en hypoxie, il hyperventile comme nous l'avons vu plus haut et ce faisant, il élimine plus de $\mathrm{CO}_{2}$ dans l'eau. Dans la phase initiale de cette hyperventilation son $\mathrm{pH}$ sanguin tend donc à augmenter. Si cette tendance n'était pas compensée, l'affinité du pigment serait également augmentée (Dejours, 1981) ce qui faciliterait la prise d'oxygène au niveau branchial mais entraverait la libération de l'oxygène au niveau tissulaire. Pour réguler l'état acide-base du sang, il existe des mécanismes métaboliques qui permettent de diminuer la concentration du système carbonate-bicarbonate sanguin et donc de corriger cette alcalinisation initiale. Chez le silure, comme chez de nombreux animaux aquatiques, ces mécanismes maintiennent le $\mathrm{pH}$ sanguin remarquablement constant dans une large gamme d'états ventilatoires et de niveaux d'oxygénation ( fig. 4).

Le bilan net de toutes ces adaptations est donc le maintien apparent de l'état d'oxygénation du sang (contenus et pressions) dans un état constant et totalement indépendant des variations extérieures. On comprend maintenant que, quel que soit $\mathrm{PO}_{2}$ dans l'eau, une augmentation ou une diminution du débit sanguin ne présenterait aucune utilité puisqu'il y a toujours autant d'oxygène dans le sang ! Et c'est là que

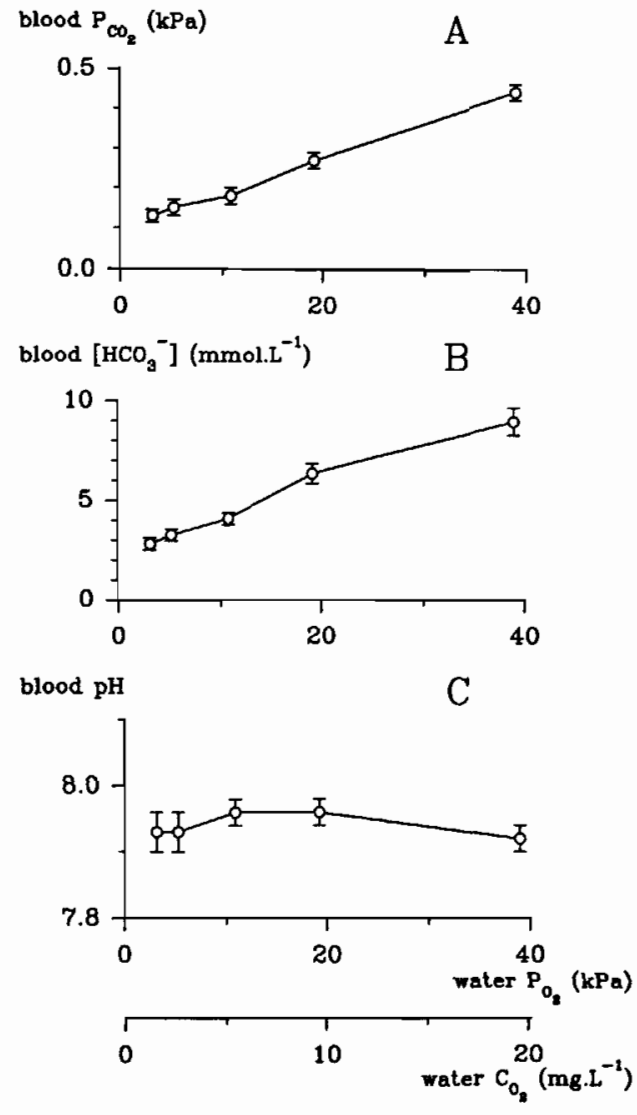

Figure 4. - Evolution dans le sang artériel de Silurus glanis: A, de la pression partielle de $\mathrm{CO}_{2}, \mathrm{Pco}_{2} ; \mathrm{B}$, de la concentration de bicarbonate, $\left[\mathrm{HCO}_{3}^{-}\right]$et $\mathbf{C}$, du $\mathrm{pH}$. Les animaux sont adaptés depuis $24 \mathrm{~h}$ à différentes valeurs de $\mathrm{Po}_{2}$ et $\mathrm{Co}_{2}$ dans l'eau inspirée ; $\mathrm{T}=13^{\circ} \mathrm{C}$ et $n=5$ à 7 animaux par point (d'après Forgue et al., 1989).

Steady-state values of blood $\mathrm{CO}_{2}$ partial pressure, $\mathrm{Pco}_{2}$ (A); bicarbonate content, $\left[\mathrm{HCO}_{3}^{-}\right]$(B) and $\mathrm{pH}$ (C) in Silurus glanis after $24 \mathrm{~h}$ exposure at selected and fixed $\mathrm{Po}_{2}$ in the inspired water. $T=13^{\circ} \mathrm{C}$ and $n=5-7$. When the inspired $\mathrm{Po}_{2}$ varies between 40 and $3 \mathrm{kPa}\left(300\right.$ to $15 \mathrm{~mm} \mathrm{Hg}$ i.e. Cwo $2=20$ and $\left.1.5 \mathrm{mg} . \mathrm{t}^{-1}\right)$, the ventilation increases (fig. $2 \mathrm{~B}$ ) and the blood $\mathrm{Pco}_{2}$ decreases. Without metabolic compensation, this could lead to a blood alkalosis. But the blood $\left[\mathrm{HCO}_{3}^{-} \mathrm{J}\right.$ increases and the net result after $24 \mathrm{~h}$ is a blood $\mathrm{pH}$ constancy (from Forgue et al., 1989).

le silure a joué un rôle particulier pour l'élaboration du concept d'homéostasie du milieu intérieur vis-à-vis de l'oxygène. Parmi les différents animaux que nous avons étudiés, c'est chez le silure que le phénomène a pu être montré de la façon la plus claire et la plus frappante. Chez l'écrevisse par exemple, la valeur moyenne de $\mathrm{Po}_{2}$ artériel ne paraît pas rester aussi rigoureusement constante bien qu'elle varie très peu. De plus, la ventilation ne maintient pas à elle seule l'homéostasie sanguine que ce soit en terme de pression ou de concentration en oxygène dans le sang artériel. On observe une augmentation du $\mathrm{pH}$ sanguin en dessous de $\mathrm{PO}_{2}$ dans l'eau $=10 \mathrm{kPa}\left(4,8 \mathrm{mg} . \mathrm{l}^{-1}\right.$ à $13^{\circ} \mathrm{C}$; ce changement de $\mathrm{pH}$ tend à augmenter l'affinité du pigment respiratoire et donc $\mathrm{Co}_{2}$ artériel ; 
Sakakibara et al., 1987) et une augmentation du débit sanguin en dessous de $5 \mathrm{kPa}\left(2,4 \mathrm{mg} \cdot \mathrm{l}^{-1}\right.$, Massabuau et Burtin, 1984).

\section{Que se passe-t-il quand la température ambiante change?}

Toutes les observations que nous venons de rapporter ont été réalisées en laboratoire à $13{ }^{\circ} \mathrm{C}$ pour faciliter l'expérimentation et le maintien du parfait état sanitaire des animaux. Dans le biotope naturel, la température est très variable et en pisciculture on utilise des températures beaucoup plus élevées. Nous n'avons pas étudié ce problème chez le silure mais la littérature présente quelques données très intéressantes pour des animaux dont les besoins en oxygène sont comparables ou même supérieures à ceux du silure. Ces données aboutissent toutes aux mêmes conclusions.

La première observation est que chez des carpes Cyprinus carpio (Ott et al., 1980) et des écrevisses Astacus leptodactylus adaptées depuis 3 semaines à 10 , 20 ou $25^{\circ} \mathrm{C}$, la consommation d'oxygène augmente mais est maintenue constante jusqu'aux mêmes pressions partielles d' $\mathrm{O}_{2}$ dans l'eau (c'est-à-dire 2-3 kPa) que chez le silure à $13^{\circ} \mathrm{C}$. Bien que ce phénomène ne soit pas très classiquement admis dans la littérature, il a sans doute une portée assez générale puisque, pour les mêmes conditions expérimentales, la pression critique est également indépendante de la température chez la truite Oncorhynchus mykiss malgré son métabolisme plus élevé: elle reste sensiblement égale à $3 \mathrm{kPa}$ $(20 \mathrm{~mm} \mathrm{Hg})$ pour des températures de 10,15 et $20^{\circ} \mathrm{C}$ (Ott et al., 1980). La deuxième observation est qu'entre 10 et $25^{\circ} \mathrm{C}$, chez Cyprinus carpio (Itazawa et Takeda, 1978; Garey, 1967) comme chez Astacus leptodactylus (Forguc, 1992), les valeurs de $\mathrm{PO}_{2}$ artériels mesurées en normoxie sont faibles indépendamment de la température. Il est donc tout à fait raisonnable de proposer que chez le silure le phénomène soit identique. En état stationnaire pour des températures variant entre 10 et $25^{\circ} \mathrm{C}$, le silure glane doit donc être parfaitement capable de maintenir sa valeur de $\mathrm{Po}_{2}$ artériel et sa consommation d'oxygène constante jusqu'à $\mathrm{Po}_{2}=$ 2-3 $\mathrm{kPa}$ dans l'eau. La solubilité de l'oxygène dans l'eau étant fonction de la température, on peut calculer qu'une valeur de $\mathrm{Po}_{2}$ critique de $3 \mathrm{kPa}$ dans l'eau correspond à $1,6 \mathrm{mg} . \mathrm{l}^{-1}$ à $10^{\circ} \mathrm{C}$ et $1,3 \mathrm{mg} . \mathrm{l}^{-1}$ à $25^{\circ} \mathrm{C}$.

Toutes ces mesures ont été faites en laboratoire et certaines conclusions peuvent sans doute surprendre, surtout quand nous montrons l'existence de principes physiologiques identiques chez des animaux physiologiquement très différents. On peut aussi se demander jusqu'à quel point ces conditions de laboratoire sont représentatives des conditions de terrain. Nous avons donc étudié des animaux dans leur milieu naturel. À l'heure actuelle nous ne savons pas faire facilement des prélèvements de sang suffisamment rapides ou totalement discrets sur des poissons placés dans leur milieu naturel. Par contre, nous disposons d'une technique qui permet de faire des prélèvements de sang artériel sur des crustacés en 15 à 30 secondes. Ce délai est suffisamment bref pour que les caractéristiques sanguines ne soient pas modifiées par la technique de prélèvement (Forgue et al., 1992). Notre laboratoire étant situé au bord du Bassin d'Arcachon, où la température varie de $6-7$ à $23-24^{\circ} \mathrm{C}$ suivant la saison, nous avons choisi deux crustacés réputés actifs

n
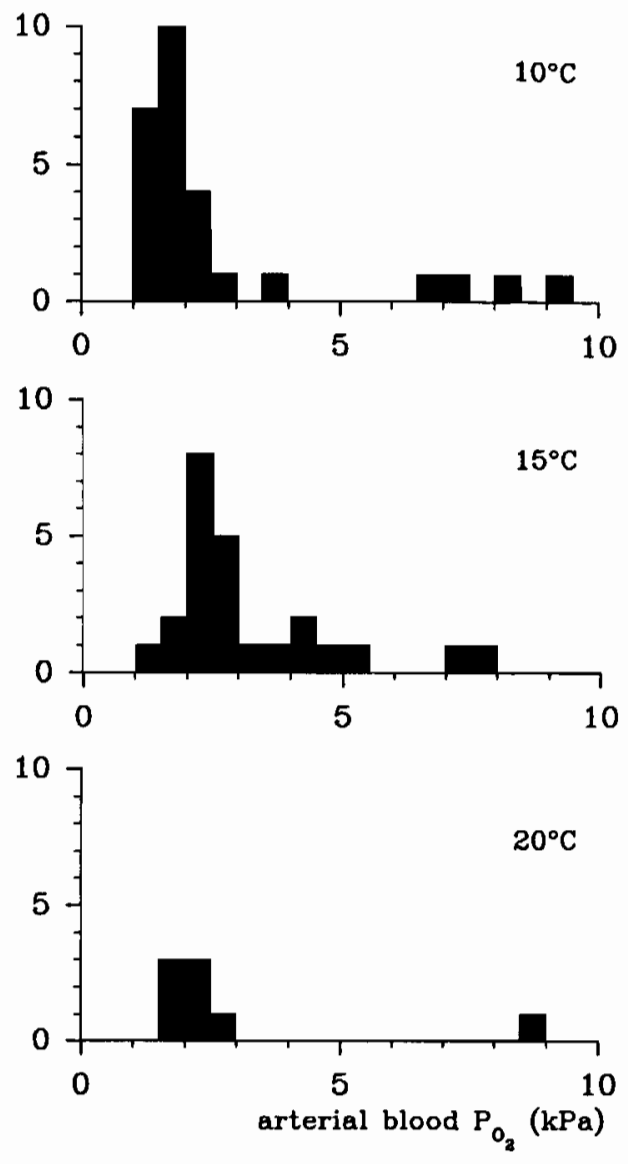

Figure 5. - Distribution des valeurs de $\mathrm{P}_{2}$ artériels chez des crabes verts Carcinus maenas étudiés dans leur milieu naturel à différentes températures et saisons. Sur le terrain comme au laboratoire, la valeur la plus fréquemment mesurée de $\mathrm{Po}_{2}$ artériel reste faible et constante quelles que soicnt la température et la saison ( $n=8$ à 25 , Massabuau et Forgue, non publié).

Arterial $\mathrm{PO}_{2}$ values in crabs Carcinus macnas studied in the field at different temperatures and seasons. Rational: the low arterial $\mathrm{PO}_{2}$ 's presented for Silurus glanis were measured at $13^{\circ} \mathrm{C}$ in laboratory conditions (fig. 2). In the laboratory at $13^{\circ} \mathrm{C}$, Carcinus maenas exhibit the same low arterial $\mathrm{Po}_{2}$ as Silurus. The frequency distributions presented here were obtained with three different groups of crabs living at different temperatures. Each animal was sampled only once ( $n$, the number of animals) and each graph presents the arterial $\mathrm{PO}_{2}$ distribution $\left(\mathrm{PaO}_{2}\right)$ for a given situation $(n=8$ to 25). Most of the values are in the low range independently of temperature and season. In carp, similar data were reported for $T$ ranging from 10 to $25^{\circ} \mathrm{C}$ (Garey, 1967; Itazawa et Takeda, 1978). We propose that the arterial $\mathrm{PO}_{2}$ in Silurus should be also independent of the temperature. 
(le crabe vert Carcinus maenas et l'étrille Necora puber) sur lesquels nous avions vérifié au laboratoire que $\mathrm{PO}_{2}$ artériel est bien maintenu à des valeurs très basses. Nous avons pu ainsi confirmer que les expériences de laboratoire avaient prédit le comportement des animaux in situ. Les valeurs de $\mathrm{Po}_{2}$ artériel chez ces animaux, dans leur milieu naturel, sont également principalement réglées à des valeurs faibles et indépendantes de la température et de la saison ( fig. 5).

Actucllement, nous travaillons au laboratoire à 13 et $23^{\circ} \mathrm{C}$ pour étudier les mécanismes physiologiques fondamentaux qui expliquent ces observations. Le problème est de comprendre comment, à $\mathrm{Po}_{2}$ constant dans le sang, on peut rendre compte d'un flux d'oxygène multiplié par un facteur $n$, de l'eau inspirée jusqu'à la cellule. Nous nous intéressons aussi aux mécanismes mis en jeu lorsque le métabolisme augmente après la prise de nourriture.

\section{CONCLUSION}

En conclusion, le silure glane est donc parfaitement capable de faire face à de très faibles niveaux d'oxygénation dans l'eau pendant des périodes d'au moins $24 \mathrm{~h}$. Toute sa stratégie d'adaptation est basée sur le maintien de $\mathrm{Po}_{2}$ dans son sang artériel à une valeur principalement faible. Sa ventilation, en augmentant quand $\mathrm{Po}_{2}$ dans l'eau diminue par exemple, lui permet de maintenir parfaitement constante cette valeur de $\mathrm{Po}_{2}$ artériel dans le sang quittant ses branchies. Le problème de l'homéostasie du milieu intérieur est donc ainsi résolu, sans changement d'état acide-base sanguin ni de débit cardiaque. Chez la carpe (Garey, 1967; Itazawa et Takeda, 1978; Takeda, 1990) comme chez les crustacés et les mollusques (Massabuau et Burtin, 1984; Massabuau et al., 1991), $\mathrm{Po}_{2}$ artériel est maintenu à des valeurs principalement faibles et constantes indépendamment des changements de $\mathrm{PO}_{2}$ dans le milieu extérieur ( fig. 6). En revanche, nous ne connaissons pas encore le comportement des poissons actifs comme les salmonidés. C'est un problème que nous pensons aborder prochainement.

Cette description de la stratégie d'adaptation des animaux aquatiques face aux changements d'oxygénation dans l'eau n'est pas classique. En effet, on voit beaucoup plus souvent dans la littérature des mesures de $\mathrm{Po}_{2}$ artériels élevées que le contraire. Nous sommes arrivés à ce résultat en créant autour de nos animaux des conditions expérimentales les moins contraignantes possibles (en utilisant des tables isolées, des systèmes de commande à distance, etc.). Ces observations de laboratoire semblent pouvoir s'appliquer aux conditions du milieu naturel puisque nous les avons vérifiées sur le terrain dans le cas de crustacés. Toutefois, il est possible que les choses se passent différemment dans un élevage intensif, si par exemple les branchies sont trop abimées ou si d'autres pathologies se développent. En tout cas, ce

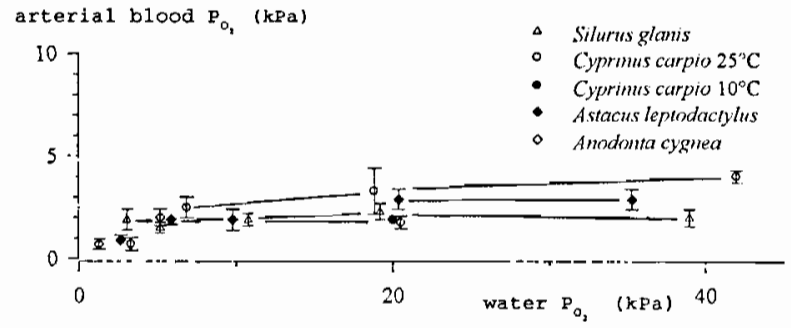

Figure 6. - Evolution de la pression partielle d' $\mathrm{O}_{2}$ (valeurs moyennes $\pm \mathrm{SE}$ ) dans le sang artériel de silures glanes. de carpe Cyprinus carpio, d'écrevisses Astacus leptodactylus ct de moules d'eau douce Anodonta cygnea maintenues à différentes $\mathrm{PO}_{2}$ dans l'eau inspirée et aussi différentes températures dans le cas de la carpe. La notion du maintien de la valeur de $\mathrm{PO}_{2}$ artériel dans une gamme très étroite - voir de la constance de $\mathrm{PO}_{2}$ artériel - que nous avons résumée chez le silure peut être retrouvéc dans chacun de ces excmples à l'exception des valeurs de $\mathrm{P}_{0_{2}}$ inspiré Ies plus basses (d'après Forgue et al., 1989; Garey, 1967; Itazawa ct Takeda, 1978; Takeda, 1990; Massabuau et Burtin, 1984; Massabuau et al., 1991).

Steady-state values of $\mathrm{O}_{2}$ partial pressures (mean values $\pm S E$ ) in the arterial blood of sheatfish Silurus glanis, carp Cyprinus carpio, crayfish Astacus leptodactylus, and freshwater mussel Anodonta cygnea kept for different periods of time at various inspired $\mathrm{Po}_{2}$ land also temperatures in the case of the carp). It is clear that to maintain a low arterial $\mathrm{Po}_{2}$, largely independent of inspired $\mathrm{Po}_{2}$ (except at the very lowest inspired $\mathrm{Po}_{2}$ ) and temperature, is not only typical of Silurus but is part of a much more general pattern distributed throughout teleosts, crustaceans and molluscs (experimental data from Forgue et al., 1989; Garey, 1967; Itazawa and Takeda, 1978; Takeda, 1990; Massabuau and Burtin, 1984; Massabuau et al., 1991).

qu'on peut d'ores et déjà garder en mémoire, ce sont les capacités maximales d'adaptation du silure glane face aux changements d'oxygénation de son milieu. Il est clair que le potentiel est très important.

\section{RÉFÉRENCES}

Dejours P. 1981. Principles of Comparative Respiratory Physiology. Elsevier/North-Holland Biomedical Press, Amsterdam, $265 \mathrm{p}$.

Forgue J. 1992. Analyse, du niveau systémique au niveau cellulaire, des mécanismes d'approvisionnement en oxygène chez les animaux aquatiques. Thèse dr. Univ. L. Pasteur, Strasbourg, $108 \mathrm{p}$.

Forgue J., J.-P. Truchot, J.-C. Massabuau 1992. Low arterial $\mathrm{Po}_{2}$ in resting crustaceans is independent of blood oxygenaffinity. J. Exp. Biol. 170, 257-264.

Forgue J., J.-C. Massabuau, J.-P. Truchot 1992. When are resting water-breathers lacking $\mathrm{O}_{2}$ ? Arterial $\mathrm{Po}_{2}$ at the anaerobic threshold in crab. Respir. Physiol. 88, 247-256.

Forgue J., B. Burtin, J.-C. Massabuau 1989. Maintenance of oxygen consumption in resting teleost Silurus glanis at various levels of oxygenation. J. Exp. Biol. 143; 305-319.

Garey W. F. 1967. Gas exchange, cardiac output and blood pressure in free swimming carp (Cyprinus carpio). Dissertation, State University of New York at Buffalo, Buffalo, NY, $123 \mathrm{p}$.

Itazawa Y., T. Takeda 1978. Gas exchange in the carp gills in normoxic and hypoxic conditions. Respir. Physiol. 35, 263-269. 
Kirsch R., G. Nonnotte 1977. Cutaneous respiration in three water teleosts. Respir. Physiol. 2), 339-354.

Le Moigne J., P. Soulier, M. Peyraud-Waitzenegger, C. Peyraud 1986. Cutaneous and gill $\mathrm{O}_{2}$ uptake in the european cel (Anguilla anguilla L.) in relation to ambient $\mathrm{PO}_{2}, 10-400$ Torr. Respir. Physiol. G.5, 341-354.

Massabuau J.-C., B. Burtin 1984. Regulation of the oxygen consumption in the crayfish Astacus leptodactylus: role of the peripheral $\mathrm{O}_{2}$ chemoreception. J. Comp. Physiol. $B$ $155,43-49$.

Massabuau J.-C., B. Burtin, M. Wheatly 1991. How is $\mathrm{O}_{2}$ consumption maintained independent of ambient oxygen in mussel Anodonta cygnea. Respir. Physiol. 83, 103-114.

Maxime V., M. Peyraud-Waitzenegger, G. Claireaux, C. Peyraud 1990. Effects of rapid transfer from sea water to fresh water on respiratory variables, blood acid-base status and $\mathrm{O}_{2}$ affinity of haemoglobin in Atlantic salmon (Salmo salar). J. Comp. Physiol. B 163, 31-39.

Nonnotte G. 1981. Cutaneous respiration in six freshwater teleosts. Comp. Biochem. Physiol. 7JA, 541-543.

Ott M. E., N. Heisler, G. R. Ultsch 1980. A re-evaluation of the relationship between temperature and the critical oxygen tension in freshwater fishes. Comp. Biochem. Physiol. 67A, 337-340.

Sakakibara Y., B. Burtin, J.-C. Massabuau 1987. Circadian rhythm of extracellular $\mathrm{pH}$ in crayfish at different levels of oxygenation. Respir. Physiol. 62, 359-367.

Takeda T. 1990. Ventilation, cardiac output and blood respiratory parameters in the carp, Cyprinus carpio, during hyperoxia. Respir. Physiol. 81, 227-240. 